\title{
Chlorpromazine reduces avoidance performance deficit in rats with dorsomedial thalamic lesions
}

\author{
KARL L. WUENSCH and LARRY W. MEANS \\ East Carolina University, Greenville, North Carolina 27834
}

\begin{abstract}
Rats with bilateral lesions of the dorsomedial area of the thalamus were shown to have a significant performance deficit on the acquisition of a one-way active shock-avoidance task relative to sham-operated controls. Administration of chlorpromazine prior to testing was shown to reduce this deficit among the DMT-lesioned animals.
\end{abstract}

Rats with lesions of the dorsomedial area of the thalamus (DMT) have been shown to be deficient in the acquisition of several appetitive tasks (Means, Harrington, \& Miller, 1975; Means, Hunt, Whiteside, \& Bates, 1973; Means, Huntley, Anderson, \& Harrell, 1973). It has been proposed that DMT-lesioned rats will be deficient on any complex task which requires them to form new associations and where stimulus conditions are changed from trial to trial (Means, Harrell, Mayo, \& Alexander, 1974). Deficits are not found in simple tasks such as approaches to food or water or runway performance (Vanderwolf, 1967).

Acquisition deficits on both one- and two-way active avoidance tasks have also been demonstrated in rats with medial thalamic damage (Delacour, 1971; Vanderwolf, 1962). Such avoidance tasks are relatively simple compared to the appetitive tasks on which DMTlesioned animals are usually shown to be deficient. The avoidance deficit is apparently not due to deficient fear conditioning, since several studies have reported normal classical fear conditioning in rats with DMT lesions (Thompson, 1963; Vanderwolf, 1963, 1969).

Vanderwolf (1971) has proposed that impaired initiation of voluntary movement accounts for the deficit shown by DMT-lesioned animals in avoidance situations. Increasing the interval between conditioned stimulus and unconditioned stimulus from 5 to $15 \mathrm{sec}$ has been shown to diminish the deficit (Vanderwolf, 1962). Compared to normal animals, cats with lesions of the dorsomedial nucleus of the thalamus have been observed to make significantly more crouching or freezing responses and fewer upward flight movements in classical fear conditioning (Roberts \& Carey, 1963). Increased frequency of freezing among medial thalamic lesioned rats placed in aversive situations such as shock avoidance has also been observed by Delacour (1971).

The purpose of the present study was an attempt to break up the freezing response in DMT-lesioned animals in a one-way active avoidance task by administration of a drug. A pilot study indicated that moderate doses of amphetamine (1.0 or $2.5 \mathrm{mg} / \mathrm{kg}$ dextro-amphetamine) did not break up freezing among DMT-lesioned rats, and higher doses $(4.0 \mathrm{mg} / \mathrm{kg})$ made the rats so hyperactive that they could not perform the task. The majority of animals run with high doses of amphetamine went into convulsions after having received only five shocks. It was decided, upon termination of this pilot study, to test the effects of a tranquilizing drug in a similar design.

\section{METHOD}

\section{Subjects and Surgical Procedures}

Subjects were 47 male Long-Evans rats approximately 160 days of age. All of the rats had previously served as subjects in an experiment investigating the effect of amphetamine upon performance of a visual-pattern discrimination in a water-filled maze. Surgery had been performed on the rats at 90 days of age. Bilateral DMT lesions were produced by passing $2.0 \mathrm{~mA}$ anodal current for $15 \mathrm{sec}$ through stainless steel electrodes stereotaxically placed $1.2 \mathrm{~mm}$ posterior to bregma, $1.0 \mathrm{~mm}$ lateral to the midline, and $6.2 \mathrm{~mm}$ ventral to the top surface of the skull. The top of the incisor bar was elevated $5.0 \mathrm{~mm}$ above interaural zero. Bilateral sham surgery consisted of lowering electrodes $5.0 \mathrm{~mm}$ into the brain at the same anterior-posterior and lateral coordinates, but passing no current. Due to an accident in the laboratory, histological data was not available for these subjects. Histological data from animals receiving almost identical surgery has been previously published (Means, Hunt, Whiteside, \& Bates, 1973; Means et al., 1975).

\section{Apparatus and Behavioral Procedures}

Twenty minutes prior to behavioral testing, each animal received an intraperitoneal injection of either physiological saline or chlorpromazine. Dosages of chlorpromazine used were $.5 \mathrm{mg} / \mathrm{kg}$ (low dose) or $1.0 \mathrm{mg} / \mathrm{kg}$ (high dose). Behavioral testing was done using a modified Lafayette Instruments one-way shock avoidance box, Model A586. The box measured $28 \times 20 \times 21 \mathrm{~cm}$ and had a $15-\mathrm{cm}$-deep escape chamber mounted along the width of one end of the box, $8 \mathrm{~cm}$ above the grid floor. A sliding panel within the escape chamber was used to push the animal back onto the grid at the end of each trial. A Grason-Stadler shock generator, Model E1064GS, was used to deliver $1.0 \mathrm{~mA}$ scrambled shock to the grid.

Each animal was individually placed on the grid floor and allowed to explore for $2 \mathrm{~min}$ with the escape chamber closed. Trial 1 started with the opening of the escape chamber. Six seconds later, shock was delivered to the grid floor. The trial ended when the animal entered the escape chamber or had received shock for a maximum of $30 \mathrm{sec}$. Each subsequent trial was started $45 \mathrm{sec}$ from the start of the previous trial. Each trial consisted of pushing the rat back onto the grid, reopening the escape chamber, and initiating shock $6 \mathrm{sec}$ into the trial. An avoidance response consisted of reentering the escape chamber before the shock was delivered. Each rat was run for 40 trials or 
Table 1

Mean Number of Errors

\begin{tabular}{crcc}
\hline & \multicolumn{3}{c}{ Drug Dosage } \\
\cline { 2 - 4 } & & \multicolumn{2}{c}{ Chlorpromazine } \\
\cline { 2 - 4 } Surgery & Saline & $.5 \mathrm{mg} / \mathrm{kg}$ & $1.0 \mathrm{mg} / \mathrm{kg}$ \\
\hline Sham & 8.7 & 7.6 & 10.2 \\
DMT & 33.3 & 20.0 & 19.3 \\
\hline
\end{tabular}

until he reached the criterion of nine avoidances on 10 consecutive trials. Number of trials to criterion and number of errors (escapes rather than avoidances) were recorded for each rat.

\section{RESULTS}

Mean numbers of errors for all groups are shown in Table 1. Trials to criterion gave a similar pattern of results.

An analysis of variance with the number of errors data (see Table 2) showed that both Lesion and Drug factors were significant $(p<.01)$, but indicated no significant interaction $(p>.05)$.

A Duncan range test was also used to compare mean number of errors between groups. Not surprisingly, all DMT groups made significantly more errors $(p<.05)$ than any sham group. No significant differences were found among the sham groups; chlorpromazine did not significantly alter shams' performances. The DMT-saline group did, however, make significantly more errors $(p<.05)$ than either the low- or high-dose lesioned animals; chlorpromazine did significantly improve avoidance performance among the DMT-lesioned animals.

\section{DISCUSSION}

That chlorpromazine causes a reduction in the avoidance performance deficit typically shown by DMT-lesioned animals suggests that such deficit may be in part due to an abnormal pattern of emotionality in such animals. It has been casually noted that DMT-lesioned rats are usually more docile and less emotional than normal rats but that, once a DMT-lesioned rat does become upset, his emotional response is of greater magnitude than that of a normal rat. In other words, DMTlesioned rats appear to have a higher threshold for emotional

Table 2

Summary Table for Analysis of Variance

\begin{tabular}{lrrrr}
\hline \multicolumn{1}{c}{ Source } & SS & df & MS & F \\
\hline Among & 4220 & 5 & & \\
Lesion & 3075 & 1 & 3075 & $59.52^{*}$ \\
Drug & 849 & 2 & 425 & $8.22^{*}$ \\
Interaction & 295 & 2 & 148 & 2.86 \\
Within & 2118 & 41 & 52 & \\
Total & 6338 & 46 & & \\
\hline
\end{tabular}

${ }^{*} p<.01$ responses, but once that threshold is crossed (by putting them in a shock-avoidance situation), the response is of great magnitude.

Normal rats usually respond to moderate levels and durations of aversive stimuli by increasing motor activity such as jumping. With repeated presentation of aversive stimuli such as shock, normal rats' response will change to freezing. Apparently DMTlesioned rats skip the first stage and show little emotional response until the stimulus conditions are so aversive or of such duration that freezing is the response made. The freezing response makes it very difficult for the animal to learn to avoid the shock by jumping to an escape platform. In passive avoidance, where the rat avoids shock by remaining in a safe area, DMT-lesioned rats do not show any performance deficit (Gerbrandt, 1965; McNew \& Thompson, 1966).

In summary, results of the present study suggest that part of the one-way active avoidance performance deficit shown by DMT-lesioned animals is due to a deviant pattern of emotionality in the shock-avoidance situation and that such deficit can be reduced by the administration of chlorpromazine.

\section{REFERENCES}

Delacour, J. Effects of medial thalamic lesions in the rat: A review and an interpretation. Neuropsychologia, 1971, 9, 157-174.

Gerbrandt, L. K. The effects of anteromedial and dorsomedial thalamic lesions on passive avoidance and activity. Psychonomic Science, 1965, 2, 29-40.

McNew, J. J., \& Thompson, R. Role of the limbic system in active and passive avoidance conditioning in the rat. Journal of Comparative and Physiological Psychology, 1966, 61, 173-180.

Means, L. W., Harrell, T. H., Mayo, E. S., \& Alexander, G. B. Effects of dorsomedial thalamic lesions on spontaneous alternation, maze activity, and runway performance in the rat. Physiology and Behavior, 1974, 12, 973-979.

Menns, L. W., Harrington, J. H., \& Miller, G. T. The effect of medial thalamic lesions on acquisition of a go, no-go, tonelight discrimination task. Bulletin of the Psychonomic Society, 1975, 5, 495-497.

Means, L. W., Hunt, M. W., Whiteside, R. R., \& Bates, T. W. Deficient acquisition and retention of single-alternation go, no-go in rats with medial thalamic lesions. Physiological Psychology, 1973, 1, 287-291.

Menns, L. W., Huntley, D. H., Anderson, H. P., \& Harrell, T. H. Deficient acquisition and retention of a visual-tactile discrimination task in rats with medial thalamic lesions. Behavioral Biology, 1973, 9, 435-450.

RoberTs, W. W., \& CAREY, R. J. Effects of dorsomedial thalamic lesions on fear in cats. Journal of Comparative and Physiological Psychology, 1963, 56, 950-958.

Thompson, $R$. Thalamic structures critical for retention of an avoidance conditioned response in rats. Journal of Comparative and Physiological Psychology, 1963, 56, 950-958.

VANDERWOLF, C. H. Medial thalamic functions in voluntary behavior. Canadian Journal of Psychology, 1962, 16, 318-330.

VANDERWOLF, C. H. The effect of medial thalamic lesions on previously established fear-motivated behavior. Canadian Journal of Psychology, 1963, 17, 183-187.

VANDERWOLF, C. H. Effects of experimental diencephalic damage on food hoarding and shock avoidance behavior in the rat. Physiology and Behavior, 1967, 2, 399-402.

VANDERWOLF, C. H. Effects of medial thalamic damage on initiation of movement and learning. Psychonomic Science, 1969, 17, 23-25.

VANDERWOLF, C. H. Limbic-diencephalic mechanisms of voluntary movement. Psychological Review, 1971, 78, 83-113. 